\title{
Myocardial ischemia reperfusion injury is alleviated by curcumin-peptide hydrogel via upregulating autophagy and protecting mitochondrial function
}

\author{
Chi-Lin Liao ${ }^{1 \dagger}$, Yang Liu ${ }^{2+}$, Meng-Zhao Huang ${ }^{1}$, Hua-Yong Liu', Zi-Liang Ye $e^{3}$ and Qiang S.a
}

\begin{abstract}
Background: Ischemia-reperfusion injury (IRI) is an important factor limiting tho uccess gol cardiac reperfusion therapy. Curcumin has a significant cardioprotective effect against IRI, cam ibj rericular remodeling induced by pressure load or Ml, and improve cardiac function. However, the poor water s bility and low bioavailability of curcumin restrict its clinical application.

Methods: In this study, we prepared and evaluated a curcumin-hydrode (cy,-hydrogel) to reduce cardiomyocyte apoptosis and reactive oxygen species formation induced by hvpoxia-re oyygenation injury, promote autophagy, and reduce mitochondrial damage by maintaining the phospm lation of Cx43.
\end{abstract}

Results: Meanwhile, cur-hydrogel can restore cardiac func n, in , bit myocardial collagen deposition and apoptosis, and activate JAK2/STAT3 pathway to alle viate myo dial ischemia-reperfusion injury in rats.

Conclusions: The purpose of this study is to el cia the rotective effects of cur-hydrogel on myocardial ischemia-reperfusion injury by regulating ap tosis, au. hagy, and mitochondrial injury in vitro and in vivo, which lays a new theoretical and experimental f unc. on for the prevention and reduction of IRI.

Keywords: Ischemia-reperfusion injy $y$, Autophag,, Apoptosis, Curcumin, Hydrogel

\section{Background}

The reperfusion therapy of actur nardial infarction can lead to more serious dysfunction after myocardial ischemia, resulting in $t^{\prime}$ e de rease of myocardial diastolic and systolic function, un ase of ventricular threshold, and the sh tening refractory period, which can be manifeste as alignant arrhythmia, cardiac insufficiency, an aven suc il death $[1,2]$. The mechanism of

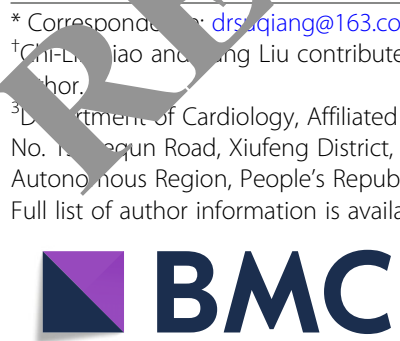

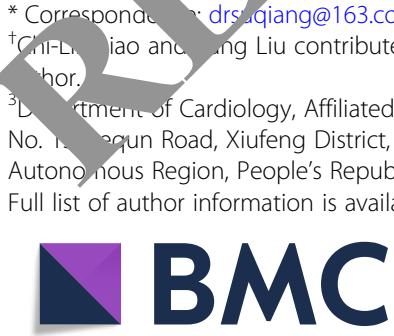

equally to this study as co-first ischemia-reperfusion injury is not completely clear. At present, the mechanisms with more evidence include oxidative stress, intracellular calcium overload, vascular endothelial injury, endoplasmic reticulum stress, and inflammatory response [3, 4]. To develop effective drugs or treatments to reduce myocardial ischemia-reperfusion injury has become a key problem to be solved urgently.

Curcumin is a polyphenolic compound extracted from the rhizome of curcuma. As an effective ingredient of traditional Chinese medicine turmeric, curcumin has a variety of pharmacological effects, such as antiinflammation, anti-oxidation, anti-apoptosis, antifibrosis, anti-tumor, cardiovascular protection, and so on. Studies have shown that curcumin has a protective

(c) The Author(s). 2021 Open Access This article is licensed under a Creative Commons Attribution 4.0 International License, which permits use, sharing, adaptation, distribution and reproduction in any medium or format, as long as you give appropriate credit to the original author(s) and the source, provide a link to the Creative Commons licence, and indicate if changes were made. The images or other third party material in this article are included in the article's Creative Commons licence, unless indicated otherwise in a credit line to the material. If material is not included in the article's Creative Commons licence and your intended use is not permitted by statutory regulation or exceeds the permitted use, you will need to obtain permission directly from the copyright holder. To view a copy of this licence, visit http://creativecommons.org/licenses/by/4.0/ The Creative Commons Public Domain Dedication waiver (http://creativecommons.org/publicdomain/zero/1.0/) applies to the data made available in this article, unless otherwise stated in a credit line to the data. 
effect on myocardial ischemia-reperfusion injury and can reduce oxidative stress injury and cardiomyocyte apoptosis [5]. Curcumin can also inhibit angiotensin II-mediated myocardial fibrosis [6] and cardiac non-benign ventricular remodeling caused by left ventricular pressure overload [7] by regulating the expression of angiotensin receptor. It can also reduce myocardial hypertrophy caused by diabetic cardiomyopathy and improve cardiac function [8]. Curcumin can also improve the cardiac function of rats after myocardial infarction in a dose-dependent manner [9] and can prevent the progression of heart failure after myocardial infarction [10]. In the treatment of heart failure, curcumin can inhibit myocardial fibrosis and inhibit the activation of myocardial fibroblasts by regulating TGF- $\beta /$ Smads signaling pathway to reduce collagen synthesis and reduce ventricular remodeling after heart failure [11]. However, curcumin has poor water solubility, easy oxidation in vitro, and low bioavailability. For example, in the human pharmacokinetic test, volunteers take a large dose of curcumin $12 \mathrm{~g}$ per day, but the blood concentration is too low to be detected, and its metabolic degradation is very fast. These shortcomings seriously restrict the wide clinical application of curcumin [12, 13].

In order to increase the water solubility and bioavailability of curcumin, various dosage forms of curcumin carriers have been developed, such as polymer nanoparticles, polymer micelles, microemulsions, micros liposomes, and polymer hydrogels [14]. There can s greatly improve the water solubility and bio a lability o curcumin by encapsulation, chemical bond hin ng, or physical adsorption. The degradation products ol small molecular hydrogels of polypeptide are amino acids, which have the advantages of easy de datio , high biocompatibility, non-toxicity, an cafety, and have been widely used in biological fielau such as threedimensional cell culture, ig co trolled release, biosensor, and so on. At rese + cu/-hydrogels are mainly made of high morecul hydrogels (such as galactose, chitosan, gelat, hitin, a d polyacrylamide gelatin) or covalently linised $\mathrm{ur}$-hydrogels [15]. It has been studied that polypeptide Nap-GFFYG-RGD and curcumin monom w..e a sembled into small molecular polypeptide hydro which significantly improved the antiimo effect, 16].

thrs ucudy, we prepared a kind of hydrogel as curcumin rier in order to improve the bioavailability of curcumin. We hypothesize that cur-hydrogel may have a better therapeutic effect on heart ischemia-reperfusion injury and explore its mechanism.

\section{Methods}

\section{Animals}

Healthy male SD rats were selected and fasted $12 \mathrm{~h}$ before the animal experiment. The rats were anesthetized by pentobarbital sodium according to their body weight. The ECG were monitored and recorded by electrocardiograph.

Cut the skin of the rat neck with aseptic scissors, fully expose the trachea, intubate the trachea with an indwelling needle, connect the small animal ventilator, giv' artificial ventilation (pressure $3 \mathrm{kpa}$, frequency 7 times/ $\mathrm{min}$ ) to assist breathing, open the chest in the lifth auxiliary room beside the sternum, separate the po dium, fully expose the heart, the left $a$ nary a tery is located between the left atrial appciclage d the pulmonary artery, and use a small cu ved needle at 1-2 mm under the left atrial appendrge. The lef anterior descending branch of the coros $v$ an, was ligatured at the upper $1 / 3$ of the $1 \mathrm{eft}$ ant $\mathrm{r}$ descending branch (note that the needl $\mathrm{a}$ th was not more than $1 \mathrm{~mm}$, and the width was not mo than $3 \mathrm{~mm}$ ). The color of the anterior war or dista end of the coronary artery was observed purplish red. ECG monitoring showed that the s egment in lead I, II, avL of ECG elevated $0.2 \quad$ as a sign of successful operation. $40 \mu \mathrm{M}$, $20 \mu \mathrm{L}$ curcu/nu or cur-hydrogel was injected to ventricular wal at the bilateral $1 \mathrm{~mm}$ of the ligation site Wh on insulin injection needle $(30 \mathrm{G})$, and then, the hest was closed and sutured. AG490 (3 mg/kg) was 1. ${ }^{c}$ ed intraperitoneally 30 min before operation in the model of heart injury. This study has been approved by the Animal Experimental Ethics Committee of Affiliated Hospital of Guilin Medical University and was carried out in accordance with the National Institutes of Health guide for the care and use of Laboratory animals (NIH Publications No. 8023, revised 1978). The rats were raised in the SPF animal room of the Clinical Research Center of the Affiliated Hospital of Guilin Medical University, feeding standard chow diet with light-dark cycle for $12 \mathrm{~h}$. After the termination of experiments, the rats were euthanized by an anesthetic overdose.

\section{Cardiac hemodynamic measurement}

A small latex balloon filled with water was inserted into the left atrium by polyethylene intubation and entered into the left ventricle. The pressure transducer was connected to measure the left ventricular diastolic pressure (LVDP), left ventricular end-diastolic pressure (LVEDP), and the maximum rate of rise and fall of left ventricular pressure. The data were input into the computer to analyze and record them.

\section{Cardiac ultrasound examination of cardiac function}

Five mice in each group were randomly selected for cardiac ultrasound 4 weeks after surgery. After intraperitoneal injection of pentobarbital sodium, the mice were fixed on a constant temperature plate at $37^{\circ} \mathrm{C}$. The chest was evenly smeared with ultrasonic coupling agent and 
detected by Mindray DP-50 ultrasound system. Each mouse was measured for 10 consecutive cardiac cycles and the left ventricular ejection fraction (LVEF) and left ventricular short axis shortening rate (LVFS), LVEF = $[($ LVEDV-LVESV $) / L V E D V] \times 100 \%, \quad$ LVFS $=[($ LVEDD LVESD)/LVEDD] $\times 100 \%$.

\section{Evans blue staining}

The coronary artery was ligated in situ, and retrograde perfusion was performed through the aorta with $2-3 \mathrm{ml}$ Evans Blue (1\%) under a certain pressure $(80 \mathrm{mmHg})$. Under the action of staining, the non-ischemic heart showed blue, which supported the display of ischemic AAR (risk zone) myocardium.

\section{$\mathrm{HE} /$ Masson staining}

The experimental animals were euthanized by injecting excessive pentobarbital sodium. The heart was removed immediately, and part of the myocardial tissue was fixed in paraformaldehyde, and ethanol was used for gradient dehydration, followed by transparent treatment and paraffin embedding. After the embedded myocardial tissue was cooled and solidified, it was cut into thin slices with a thickness of $5 \mu \mathrm{m}$. The myocardial tissue of rats was stained with HE (hematoxylin-eosin staining) and collagen was stained with Masson.

Determination of biochemical indexes of myoca.uial tissue

The myocardial tissue was homogenized at 60 , for 60

$\mathrm{s}$ for 2 times in an automatic samp grinder, as d the supernatant was centrifuged at $3500 / \mathrm{min}$ for $10 \mathrm{~min}$ at $4{ }^{\circ} \mathrm{C}$. The samples were separated an tored in the refrigerator at $-80{ }^{\circ} \mathrm{C}$ for the cormination of content and the activities of SOD, CA - , GPS,GR, respectively. After strictly foll o the instructions of the kit, the OD values we det mined by enzyme labeling instrument.

\section{Enzyme colorinetr nethod}

The acti-ities of Ca -ATPase and $\mathrm{Na}^{+}-\mathrm{K}^{+}$-ATPase in aorta $m$ ter nined by enzyme colorimetry (Nanjing Jiar teng titute of Biological Engineering, China). rict according to the instructions of ultra-trace $\mathrm{Ca}^{2+}$ A ase and $\mathrm{Na}^{+}-\mathrm{K}^{+}$-ATPase detection kit, the activities of $y$ ine in mocardial tissue homogenate were determined.

\section{Preparation of cur-hydrogel}

The peptide RADA16-I (Ac-RADARADARADARADA$\mathrm{CONH} 2$ ) stored at $4{ }^{\circ} \mathrm{C}$ and was synthesized by Shanghai Bootech BioScience \& Technology (Shanghai, China).

The method of configuration of curcumin-RADA16-I solution was as follows: appropriate curcumin and
RADA16-I were placed in $10 \mathrm{ml}$ vials, and water was added to vials, obtaining curcumin solution with concentration of $5.0 \times 10^{3} \mathrm{M}$ and RADA16-I solution with concentration of $5.8 \times 10^{5} \mathrm{M}(0.1 \mathrm{mg} / \mathrm{ml})$. The solution is left in a mixing pan for about 5 days.

\section{Dynamic light scattering (DLS)}

The particle size distribution of the cur-hydro 'suspension was measured by dynamic light/scatterin ticle size analyzer (Nano-ZS90, Man १, UK') The suspension is shaken vigorously beforc mea rement.

\section{Drug release kinetics in vitro}

Under the simulated env nme. of physiological temperature in vitro, the release me and rate of curcumin from co-assemb ea drogel were observed. It was detected by high-perform. e liquid chromatographymass spectromfry C-MS)

\section{Hypoxia-reoxygen, on model of cardiomyocytes}

H9C2 ca mvocyes (Boster Biological Technology, Wuhan, Chira) were cultured in DMEM medium containing $10 \%$ serum (GIBCO, USA) and $1 \%$ penicillinSt omycin at $37{ }^{\circ} \mathrm{C}$ and $5 \% \mathrm{CO}_{2}$ incubator. According to th experimental group, the original complete culture diam was replaced by serum-free low-sugar DMEM midium, and $\mathrm{H} 9 \mathrm{C} 2$ cardiomyocytes were placed in an anoxic incubator with $37{ }^{\circ} \mathrm{C}, 5 \% \mathrm{CO}_{2}$, and $2 \% \mathrm{O}_{2}$ for 12 h. After hypoxia, the culture medium was replaced by complete culture medium and reoxygenated in an incubator of $37^{\circ} \mathrm{C}$ and $5 \% \mathrm{CO}_{2}$ for $12 \mathrm{~h}$ to establish a cell HR model.

\section{MTT assay}

H9C2 cells were plated in 96-well plates and we used MTT assay to detect the cell viability. MTT $(0.5 \mathrm{mg} / \mathrm{mL}$, Beyotime Biotechnology, China) was added after curcumin treatment and incubated for $3 \mathrm{~h}$ at $37^{\circ} \mathrm{C}$. And $150 \mu \mathrm{L}$ DMSO was added and incubated for $15 \mathrm{~min}$. We measured the absorbance at $490 \mathrm{~nm}$.

\section{Flow cytometry}

The Annexin V-FITC/PI apoptosis detection kit was purchased from Solebao Company (Beijing, China), and an appropriate amount of logarithmic growth phase cells were washed twice with pre-cooled PBS. The cells were suspended with $500 \mathrm{ul}$ of bound buffer, mixed with $5 \mu \mathrm{l}$ of annexin V-FITC and PI, respectively, and placed at room temperature for $15 \mathrm{~min}$. The apoptosis rate was determined by flow cytometry.

\section{Single fluorescent GFP-LC3 plasmid transfection}

After transient transfection of GFP-LC3 plasmid into H9C2 cells, the changes of green bright spots of GFP- 
LC3 in each group were observed under double fluorescence microscope, and 5 visual fields were randomly selected in each experimental group to take pictures.

\section{TUNEL staining}

According to the instructions, the cells or tissues were successively added with biotin labeling solution and 3,3diaminobenzidine carbon tetrachloride (DAB) chromogenic solution and used PBS. Finally, the 3DHISTECH Pannoramic SCAN system was used to scan 5 nonoverlapping visual fields in each group. The apoptotic nuclei and the total number of apoptotic nuclei in the visual field were calculated by Image J software. The apoptosis rate of cardiomyocytes $=$ the number of apoptotic nuclei/the total number of nuclei $\times 100 \%$.

\section{DCFH-DA probe staining}

DCFH-DA probe (Sigma, USA) is used to detect the formation of reactive oxygen species. The cardiomyocyte culture medium was removed; diluted DCFH-DA (10uM) probe $1 \mathrm{ml}$ was added and incubated at $37^{\circ} \mathrm{C}$ in $5 \% \mathrm{CO}_{2}$ incubator for $20 \mathrm{~min}$. Rinse with PBS for 3 times $\times 1$ min to fully remove the DCFH-DA probe that did not enter the cell. Then, the fluorescence intensity was detected by laser confocal microscope.

\section{Determination of mitochondrial membrane potent}

JC-1 reagent (T3168) was purchased from Ser $1 \mathrm{~d}$. F Technology Co., Ltd. (China). Strictly folloy instruc tions. $1 \mu \mathrm{g} / \mathrm{mL} \mathrm{JC-1}$ working solution was prep d and incubated at $37^{\circ} \mathrm{C}$ for $20 \mathrm{~min}$, PBS Five visual tields were selected for each group and 1 otographed under confocal microscope.

\section{Western blot}

Take appropriate amov f cell in logarithmic growth phase, after RIPA $c^{\prime}$ vag oxtract total protein, BCA method. After cuan ative denaturation, protein electrophoresic mbrane transfer-closure-I antiincubation-II an incubation-development exposure were carried out acc,rding to the operation steps. The express the target protein was expressed by the ratio the g value.

\section{S. sticar analysis}

All $\alpha$ is presented as a mean \pm S.E.M. Statistical analysis was performed using a one-way ANOVA. $P$ value < 0.05 was considered as statistically significant.

\section{Results}

\section{Construction and characterization of cur-hydrogel}

cur-hydrogel can form nanofibers with different thickness, the diameter is about $1000 \mathrm{~nm}$ (Fig. 1a), and interweave and winding each other to form a three- dimensional network structure. The cur-hydrogel has good mechanical properties and stability and can slowly release curcumin monomer in vitro (Fig. 1b).

\section{The protective effect of cur-hydrogel on cardiomyocyte} injury induced by HR was better than curcumin We treated the cells with different concentr tions of curcumin or curcumin-loaded hydrogels $\left(20,40, \mathrm{C}_{\mathrm{M}}\right)$ for $24 \mathrm{~h}$, followed by hypoxia-reoxygenation injury. T/ results showed that both curcun grou and curcumin-loaded hydrogel group hau int tory effect on cell death, and the effect of $c$ (r-hydrogel group was better at the same concentration ( $2 \mathrm{a})$.)

We chose $40 \mu \mathrm{M}$ curcumin roup urcumin-loaded hydrogel group to inhib cell a h. The results of flow cytometry (Fig. 2b) a TUNњL staining (Fig. 2c) showed that cur-hvdrogel ld reduce apoptosis better at the same corcen ation. The results of Western blotting showed tha $\mathrm{A}$ or curcumin-loaded hydrogel decreased the pro in levels of Bid, Bax, caspase-3, and caspase- 9 ced by hypoxia-reoxygenation, and the effect of cui-bya $\mathrm{y}_{\mathrm{j}}$ gel was better than that of curcumin group (Fig. ?d).

hown in Fig. 3a, both curcumin and cur-hydrogels can 1 ibit cell ROS production, and the effect of curIrogel is better at the same concentration. The changes of p38 MAPK/NF-kB in ROS-related pathway were determined by Western blotting. Hypoxiareoxygenation injury increased the expression of pamp T-p38 MAPK and NF-kB, while cur-hydrogel significantly decreased the expression of these proteins (Fig. 3b).

Curcumin has been reported to affect the level of autophagy. In this study, mRFP-GFP-LC3 adeno-associated virus was used to transfect $\mathrm{H} 9 \mathrm{C} 2$ cardiomyocytes labeled LC3. As shown in Fig. 4a, the expression of autophagosomes decreased due to hypoxia-reoxygenation injury, and cur-hydrogel could promote autophagy more than curcumin. In Fig. 4b, the transformation of autophagy marker protein LC3-I to LC3-II was further detected by western blot, and the level of p62 protein was measured. Compared with curcumin, cur-hydrogel could promote the transformation from LC3-I to LC3-II and inhibit the expression of $\mathrm{p} 62$.

We used JC-1 probe to detect the degree of mitochondrial depolarization after cur-hydrogel treatment. In the HR group, the mitochondrial membrane potential decreased (red fluorescence decreased). After curcumin treatment, the decrease of mitochondrial membrane potential was alleviated, and after cur-Hydrogel treatment, the decrease of mitochondrial membrane potential was further alleviated. Hydrogel had no effect on mitochondrial membrane potential as in the control group (Fig. 5a). Western blotting showed that p-Cx43/Cx43 
A

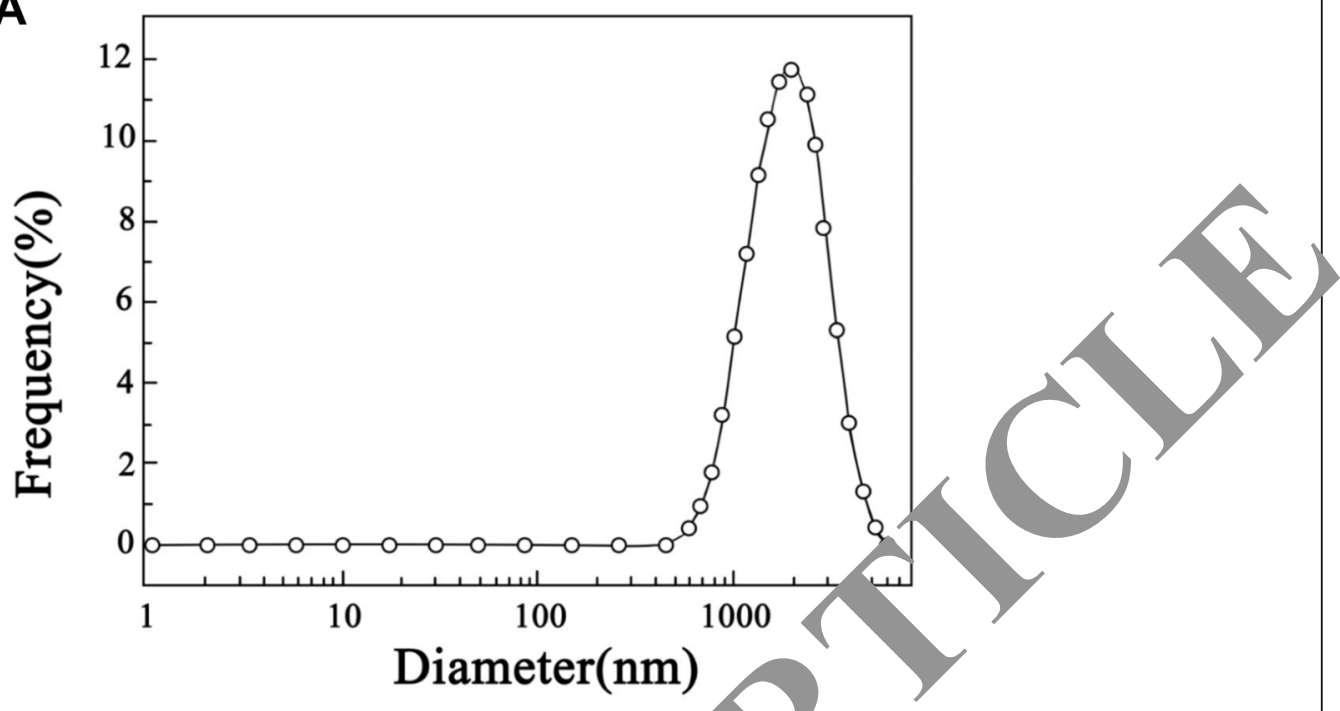

B

Fig. 1 Characteristics of cur-hydrogel. a Dro used to detect the particle size of curcumin hydrosol. $\mathbf{b}$ The cumulative release amount of curcumin from hydrogel to polution $\left(37^{\circ} \mathrm{C} \mathrm{pH} 7.4\right)$

ratio was decreas a by treatment, $\mathrm{p}-\mathrm{Cx} 43 / \mathrm{Cx} 43$ ratio was improved/a urcumı treatment, and p-Cx43/Cx43 ratio was furthe improved by cur-hydrogel group (Fig. 5b)

cur drog va, superior to curcumin in relieving

\section{yoc dial is nemia-reperfusion injury in rats}

h. estawished a rat model of myocardial ischemiarepe. sion and treated with curcumin or hydrogel curcumin. First of all, we measured the left ventricular diastolic blood pressure (LVDP), left ventricular enddiastolic pressure (LVEDP), and the maximum rate of rise and fall of left ventricular pressure (dp/dt max). The results showed that cur-hydrogel could significantly restore the cardiac function of model mice, and its effect was better than that of curcumin. (Table 1). Superoxide dismutase (SOD), catalase (CAT), glutathione peroxidase
(GPX), and glutathione reductase (GR) coordinate to prevent excessive level of reactive oxygen species in cells, which is collectively referred to as protective enzyme system. cur-hydrogel can increase the activity of these enzymes more than curcumin and play a protective role in cardiomyocytes (Table 2). The activities of $\mathrm{Ca}^{2+}$. ATPase and $\mathrm{Na}^{+}-\mathrm{K}^{+}$-ATPase in myocardial sarcoplasmic reticulum were determined. cur-hydrogel could significantly restore the activity of $\mathrm{Ca}^{2+}$-ATPase and $\mathrm{Na}^{+}-\mathrm{K}^{+}$ATPase in model mice (Table 3 ). The hydrogel treatment had no significant effect on the cardiac function of rats. However, with JAK2/STAT3 inhibitor AG490, the effect of cur-hydrogel was reversed. (Tables 1, 2, and 3).

Left ventricular ejection fraction (EF) and left ventricular shortening fraction (FS) were detected by echocardiography. The results showed that cur-hydrogel could restore the cardiac function of model rats (Fig. 6a, b). 


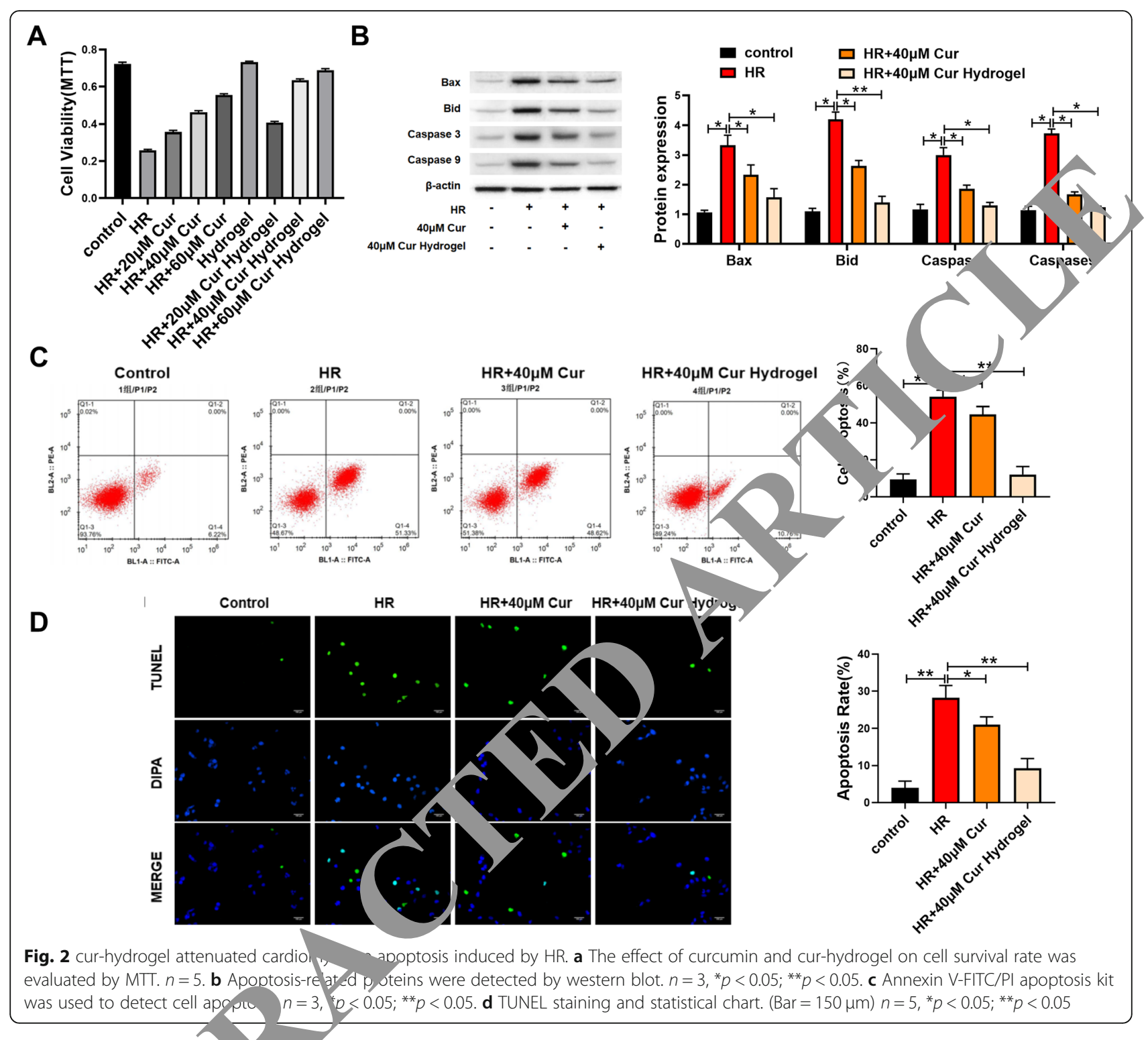

The results of $\mathrm{F}_{\mathrm{S}} \mathrm{S}$ blue st, ning showed that cur-hydrogel reduced the area on vocardial infarction (Fig. 6c). Treated with AG990, the EF a) d FS was decreased (Fig. 6a, b), and the area $m_{\text {, }}$ ca dial infarction was increased (Fig. 6c).

Tl myo dial tissue of rats was stained with HE and llag $n$ was stained with Masson. The results showed th. cur r.ydrogel could restore the orderly arrangement of c. \%myocytes and inhibit the excessive deposition of collagen, AG490 blocked the therapeutic effect of curcumin hydrogel (Fig. 6d).

Tissue TUNEL staining showed that, consistent with the results in vitro, heart injury led to the increase of apoptosis, curcumin could reduce the occurrence of apoptosis to some extent, but cur-hydrogel had better effect. However, AG490 promoted apoptosis of rat heart cells (Fig. 6e).
JAK2/STAT3 is an important intracellular signal transduction pathway, which involves a variety of pathological and physiological processes such as apoptosis, cell proliferation and differentiation. Western blot detection of myocardial homogenate showed that cur-hydrogel activated JAK2/STAT3 signal pathway in rat cardiomyocytes and played a role in myocardial protection. After blocking the JAK2/STAT3 pathway with AG490, the therapeutic effect of curcumin hydrogel disappeared (Fig. 6f). The above results showed that the effect of cur-hydrogel was better than that of curcumin.

\section{Discussion}

Cardiac rational ventricular remodeling caused by myocardial infarction is one of the most common clinical causes of heart failure, which accelerates researchers' 

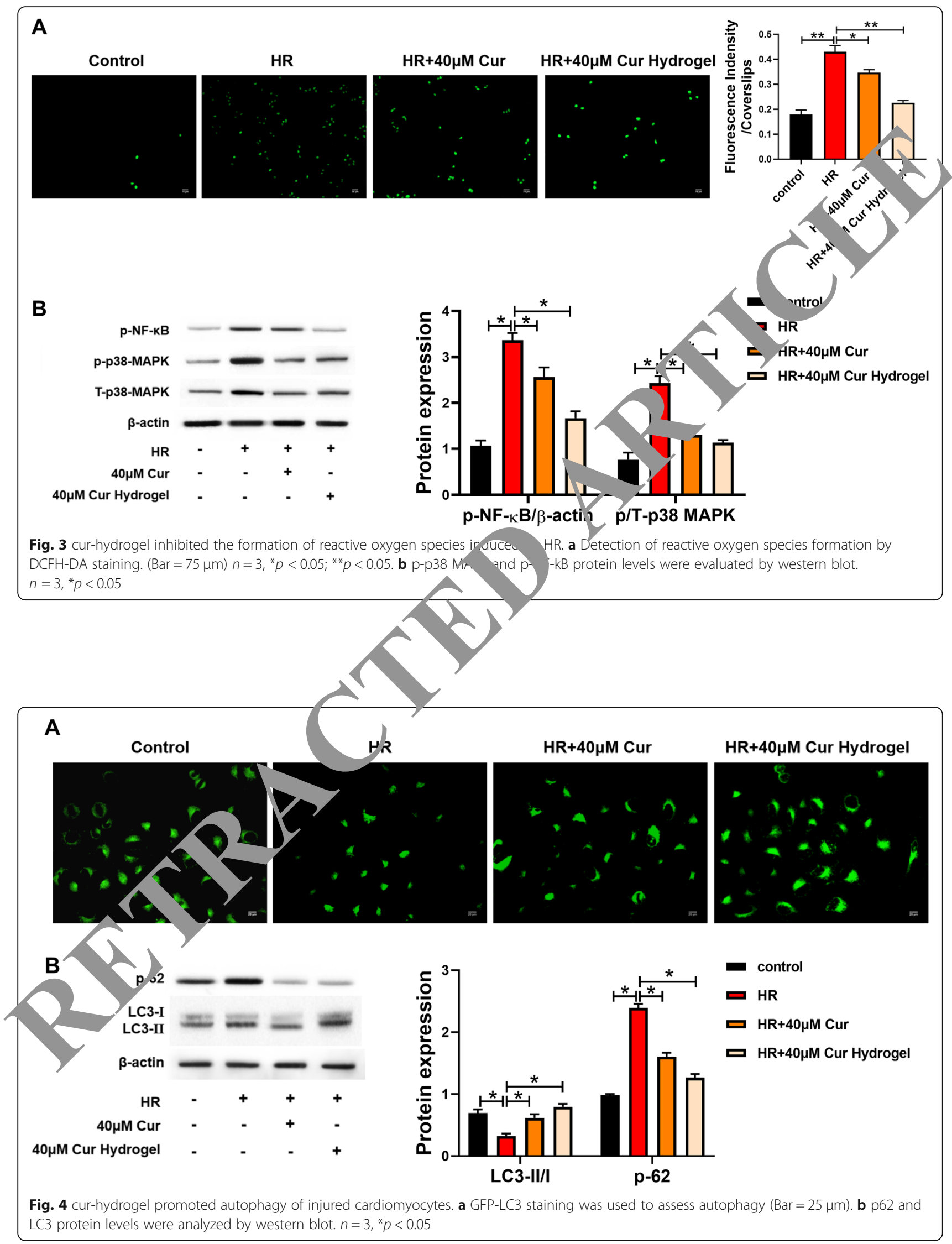


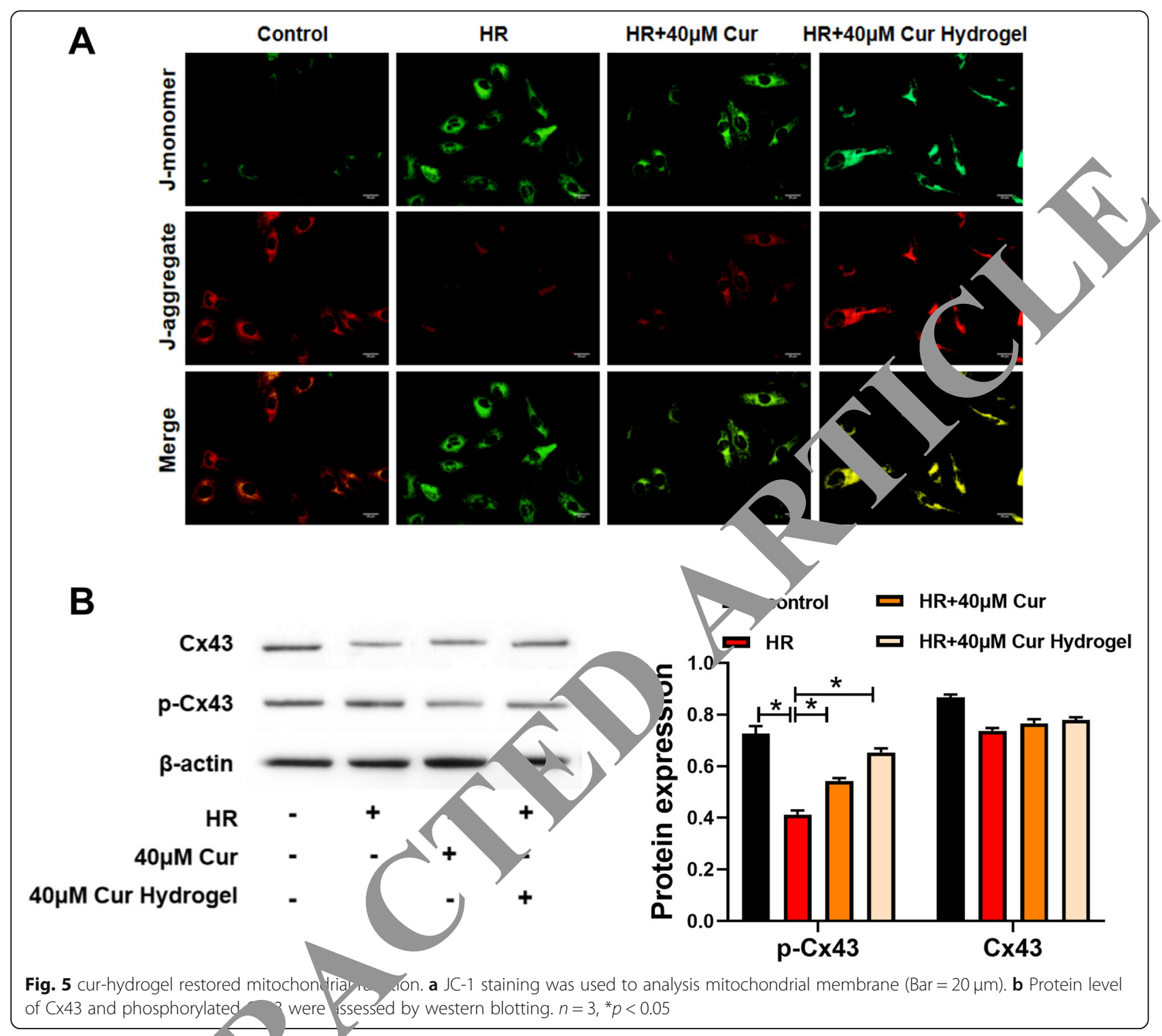

efforts to develop new ttments and techniques to inhibit ventricul emodeli, $\delta$ after myocardial infarction [17]. Among all k 's of biomaterials, injectable hydrogel has the advantagy, of good biocompatibility, degradability, h rater solubility, and injectability, so it has a good development prospect and research value in the repair and treatment of myocardial infarction. Simple injection of hydrogel can provide mechanical support and structural filling for the damaged cardiac structure; increase the thickness of the ventricular wall in the

\begin{tabular}{|c|c|c|c|c|}
\hline Grou & LVDP $(\mathrm{mmHg})$ & LVEDP $(\mathrm{mmHg})$ & $+\mathrm{dp} / \mathrm{dtmax}(\mathrm{mmHg} / \mathrm{s})$ & $+\mathrm{dp} / \mathrm{dtmin}(\mathrm{mmHg} / \mathrm{s})$ \\
\hline Sham & $98.26 \pm 8.59$ & $1725 \pm 15.36$ & $1802.45 \pm 157.24$ & $1326.57 \pm 100.78$ \\
\hline IR & $61.52 \pm 25.92 *$ & $1043.26 \pm 98.25 *$ & $1236.26 \pm 118.46$ * & $862.34 \pm 56.08 *$ \\
\hline IR+40 $\mu \mathrm{M}$ Cur & $74.25 \pm 16.94$ \# & $1291.05 \pm 113.05$ \# & $1369.34 \pm 105.34$ \# & $969.78 \pm 87.49^{\#}$ \\
\hline IR+40 $\mu \mathrm{M}$ Cur Hydrogel & $81.93 \pm 8.33^{\#}$ & $1481.23 \pm 122.45$ \# & $1527.57 \pm 144.28{ }^{\#}$ & $1149.78 \pm 94.26{ }^{\#}$ \\
\hline IR+40 Hydrogel & $65.68 \pm 18.911^{N S}$ & $1108.32 \pm 101.35^{N S}$ & $1271.34 \pm 101.76^{N S}$ & $871.89 \pm 76.32^{N S}$ \\
\hline IR+40 $\mu$ MCur Hydrogel+AG490 & $63.74 \pm 22.45^{N S}$ & $1089.34 \pm 110.15^{N S}$ & $1289.67 \pm 121.54$ NS & $898.23 \pm 85.12^{N S}$ \\
\hline
\end{tabular}

${ }^{*} p<0.05$ vs sham; $\# p<0.05$ vs IR; $n s$ nonsignificant 
Table 2 Detection of biochemical indexes in myocardial tissues

\begin{tabular}{|c|c|c|c|c|}
\hline Group & SOD & CAT & GPX & $\mathbf{R}$ \\
\hline Sham & $153.86 \pm 13.67$ & 3.79 & $67.39 \pm 4.55$ & 51.42 \\
\hline $\mathbf{R}$ & $48.38 \pm 25.49 *$ & $3.45 \pm 1.72 *$ & $3.29 \pm 4.43 *$ & 15.83 \\
\hline $2+40 \mu \mathrm{M}$ Cur & $94.25 \pm 8.88^{\#}$ & $22.15 \pm 2.62 \#$ & $44.37 \pm 3.86 \#$ & $39.58 \pm 13$ \\
\hline R+40 $\mu \mathrm{MCur}$ Hydrogel & $135.43 \pm 11.35 \#$ & $38.93 \pm 3.06^{\#}$ & $52.98 \pm 4.93{ }^{\#}$ & \\
\hline R+40 Hydrogel & $50.21 \pm 32.16^{N S}$ & $4.76 \pm 1.87^{N S}$ & $24.18 \pm 2.76^{N S}$ & \\
\hline R+40 $\mathrm{MMCur}$ Hydrogel+AG490 & $61.42 \pm 21.89^{N S}$ & $16.31 \pm 2.90^{N S}$ & $27.37 \pm 4.98^{N S}$ & \\
\hline \multicolumn{5}{|c|}{$p<0.05$ vs sham; $\# p<0.05$ vs IR; $n s$ nonsignificant } \\
\hline \multicolumn{2}{|c|}{$\begin{array}{l}\text { infarcted area to prevent the ventricular wall from grad- } \\
\text { ually thinning, dilating, or even rupturing; and improve } \\
\text { cardiac function [18]. It can also be used as a carrier for } \\
\text { carrying cytokines such as TIMP-3, VEGF, or FGF-2 and } \\
\text { drug delivery }[19,20] \text {. } \\
\text { Curcumin is an effective ingredient of turmeric, a trad- } \\
\text { itional Chinese medicine, and has been used in China } \\
\text { for thousands of years. Current studies have shown that } \\
\text { it has a good protective effect on cardiovascular diseases, } \\
\text { including diabetic cardiomyopathy [21], hypertension } \\
\text { [22], cardiac hypertrophy [23], myocardial ischemia/re- } \\
\text { perfusion [24], and heart failure [25]. In addition, curcu- } \\
\text { min inhibits the progression of cardiomyocyte } \\
\text { hypertrophy, apoptosis, and fibrosis by reducing inflam- } \\
\text { mation and oxidative damage in cardiomyocytes and } \\
\text { general heart tissue [26]. Curcumin is a known P } \\
\text { hibitor, in the rat model of diabetic cardiomyopathy, } \\
\text { curcumin can improve cardiac contractile ction by } \\
\text { inhibiting cardiomyocyte hypertrophy and cara. }\end{array}$} & \multicolumn{3}{|c|}{ 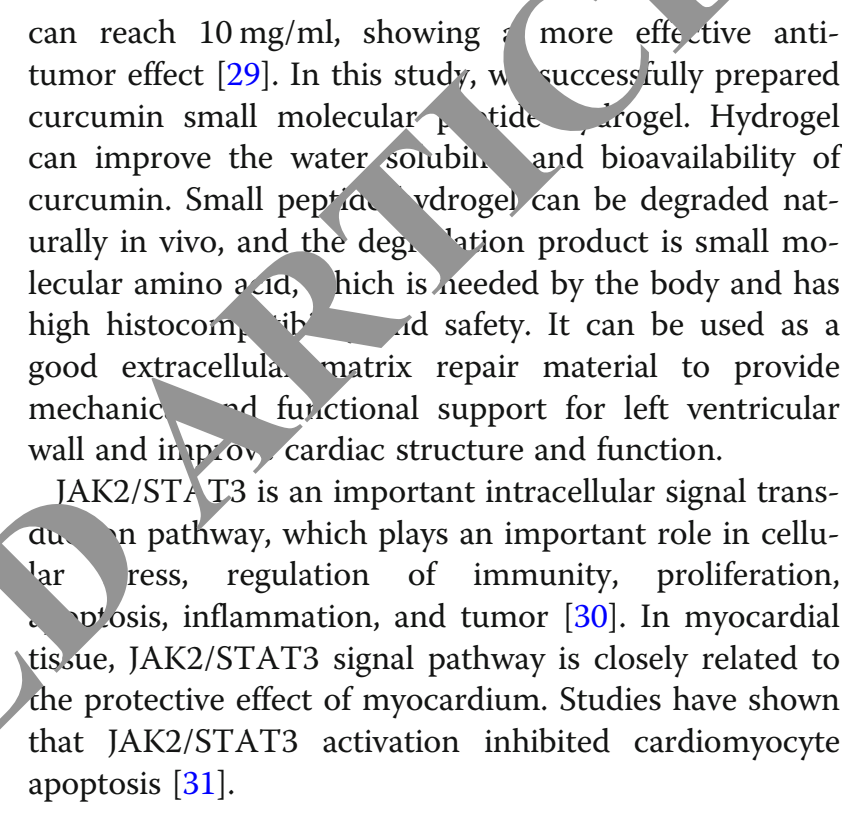 } \\
\hline
\end{tabular}
sis and reduce the expression of TGF $\beta 1$ [27]. Or, 1 curcumin can reduce the progression o myocarcial fibrosis induced by angiotensin II in rats, an his protective effect is related to the decrease ${ }^{\mathrm{T}} \mathrm{GF}-\beta 1$ expression and smad2/3 phosphorylation level in heart [28]. This study further confirms at curcumin released from hydrogel can reduce he rmation of reactive oxygen species, restore mitach Trial runction, improve cardiac function, inhi myocal al apoptosis, and activate JAK2/STAT3 path v.

Previoys studies h, ve shown that curcumin is linked to the ti te to form compound I, which can greatly imr ve is water solubility, and the solubility in PBS

\section{Conclusions}

In general, our study showed that cur-hydrogel could reduce cardiomyocyte apoptosis, ROS formation and mitochondrial damage in vitro, and the effect was better than that of curcumin. Compared with curcumin, curhydrogel can effectively improve cardiac function (FS, $\mathrm{EF}$ ), inhibit left ventricular dilatation, inhibit ventricular remodeling and collagen synthesis, and activate JAK2/ STAT3 pathway to protect injured myocardium in rat ischemia-reperfusion model, which may become a feasible scheme for clinical treatment of myocardial infarction.

\begin{tabular}{|c|c|c|}
\hline Grou, $Y$ & $\mathrm{Ca}^{2+}$ ATPase $\left(\mu \mathrm{mol} \mathrm{mg}^{-1} \mathrm{~h}^{-1}\right)$ & $\mathrm{Na}^{+}-\mathrm{k}^{+}$-ATPase $\left(\mu \mathrm{mol} \mathrm{mg}^{-1} \mathrm{~h}^{-1}\right)$ \\
\hline Sham & $1.65 \pm 0.14$ & $3.28 \pm 0.25$ \\
\hline IR & $0.594 \pm 0.05 *$ & $1.274 \pm 0.15 *$ \\
\hline $\mathrm{IR}+40 \mu \mathrm{M}$ Cur & $0.874 \pm 0.077^{\#}$ & $2.064 \pm 0.19^{\#}$ \\
\hline IR+40 $\mathrm{MMCur}$ Hydrogel & $1.214 \pm 0.11^{\#}$ & 2. $664 \pm 0.25 \#$ \\
\hline IR+40 Hydrogel & $0.601 \pm 0.09^{N S}$ & $1.358 \pm 0.23^{N S}$ \\
\hline IR+40 $\mathrm{MMCur}$ Hydrogel+AG490 & $0.681 \pm 0.11^{N S}$ & $1.562 \pm 0.14^{N S}$ \\
\hline
\end{tabular}

${ }^{*} p<0.05$ vs sham; $\# p<0.05$ vs IR; $n s$ nonsignificant 


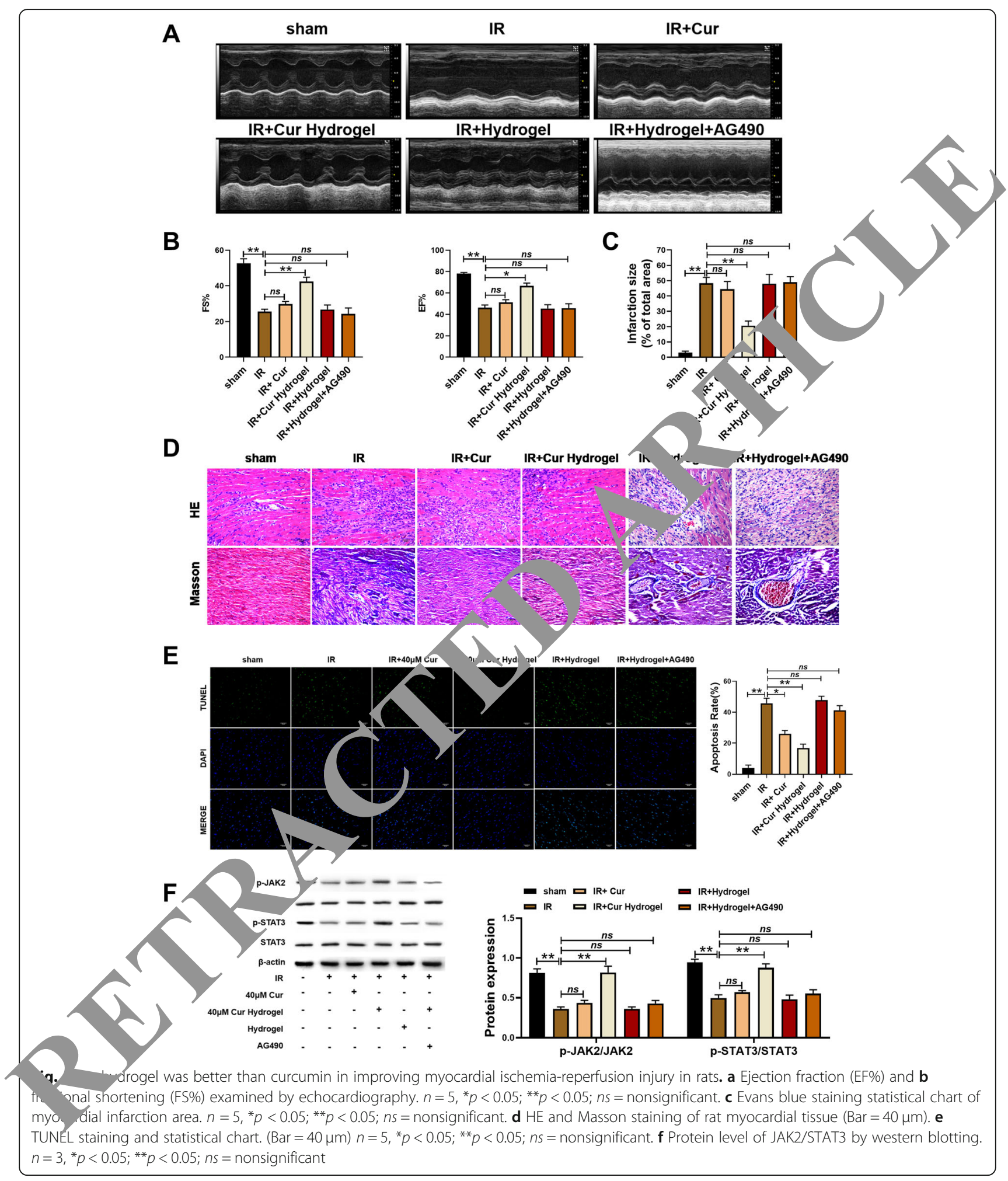




\section{Abbreviations}

IRI: Ischemia-reperfusion injury; cur-hydrogel: Curcumin-hydrogel; LCMS: Liquid chromatography-mass spectrometry; SOD: Superoxide dismutase; CAT: Catalase; GPX: Glutathione peroxidase; GR: Glutathione reductase

\section{Acknowledgements}

Not applicable.

\section{Authors' contributions}

Chi-Lin Liao and Zi-Liang Ye performed the majority of experiments and analyzed the data; Yang Liu performed the molecular investigations; Meng-Zhao Huang and Qiang Su designed and coordinated the research; Hua-Yong Liu wrote the paper. The authors read and approved the final manuscript.

\section{Funding}

The authors received no funding for this work.

\section{Availability of data and materials}

The datasets used and analyzed during the current study are available from the corresponding author on reasonable request.

\section{Ethics approval and consent to participate}

Not applicable.

\section{Consent for publication}

Not applicable.

\section{Competing interests}

The authors declare that they have no competing interests.

\section{Author details}

'Department of Cardiology, People's Hospital of Baise, Baise 533000, People's Republic of China. ${ }^{2}$ Department of Cardiology, The Second People's Hospital of Nanning City, The Third Affiliated Hospital of Guangxi Medical Un Nanning 530031, People's Republic of China. ${ }^{3}$ Department of Carliolo Affiliated Hospital of Guilin Medical University, No. 15, Lequn P rad, Xiufer District, Guilin 541001, Guangxi Zhuang Autonomous Regicn, Dle's Republic of China.

\section{Received: 30 July 2020 Accepted: 14 December 020} Published online: 28 January 2021

\section{References}

1. Ibanez B, Heusch G, Ovize M, Van de Wer mang therapies for myocardial ischemia/reperfusion injury. J Am Coll Carciol/201s, o5(14):1454-71.

2. Vogel B, Mehta SR, Mehran D... rfusion trategies in acute myocardial infarction and multivesse' 'isease Nat Rev ardiol. 2017;14(11):665-78.

3. Liu Y, Xu J, Wu M, Kang L, a cellor cells and cellular mediators of immune system in olved in a cinflammation and fibrosis after myocardial infar J Cell Phys, 2020;235(12):8996-9004.

4. Yang Y, Zhou Gao hen L, Li L. Endoplasmic reticulum stress and focused drug disc Nov in cardio Mar disease. Clin Chim Acta. 2020;504:125-37.

5. Duan , Yang Y, Yan J, Ya S, Liu J, Zhou J, et al. The effects of curcumin postof the JA, TAT3 signaling pathway. Basic Res Cardiol. 2012;107(3):263. XF, Zho. LH, Bai F, Wang NP, ljaz Shah A, Garner R, et al. Dual ACEand angiotensin II AT1 receptor antagonism with curcumin maladaptive cardiac repair and improve ventricular systolic function mayocardial infarctionin rat heart. Eur J Pharmacol. 2015;746:22-30.

7. Ya, QH, Wang DQ, Cui CC, Yuan ZY, Chen SB, Yao XW, et al. Curcumin ameliorates left ventricular function in rabbits with pressure overload: inhibition of the remodeling of the left ventricular collagen network associated with suppression of myocardial tumor necrosis factor-alpha and matrix metalloproteinase-2 expression. Biol Pharm Bull. 2004;27(2):198-202.

8. Yu W, Wu J, Cai F, Xiang J, Zha W, Fan D, et al. Curcumin alleviates diabetic cardiomyopathy in experimental diabetic rats. PLoS One. 2012;7(12):e52013.

9. Sunagawa Y, Sono S, Katanasaka Y, Funamoto M, Hirano S, Miyazaki Y, et al. Optimal dose-setting study of curcumin for improvement of left ventricular systolic function after myocardial infarction in rats. J Pharmacol Sci. 2014; 126(4):329-36
10. Saeidinia A, Keihanian F, Butler AE, Bagheri RK, Atkin SL, Sahebkar A Curcumin in heart failure: a choice for complementary therapy? Pharmacol Res. 2018;131:112-9.

11. Gorabi AM, Hajighasemi S, Kiaie N, Rosano GMC, Sathyapalan T, Al-Rasadi K, et al. Anti-fibrotic effects of curcumin and some of its analogues in the heart. Heart Fail Rev. 2020;25(5):731-43.

12. Wahlstrom B, Blennow G. A study on the fate of curcumin in the rat. Acta Pharmacol Toxicol (Copenh). 1978;43(2):86-92.

13. Peng JR, Qian ZY. Drug delivery systems for overcoming the bi availiability of curcumin: not only the nanoparticle matters. Nanomedicine 2014 9(6):747-50.

14. Hatcher H, Planalp R, Cho J, Torti FM, Torti SV. Curcur (n: from ancien medicine to current clinical trials. Cell Mol Life Sci.zo $5(11): 163-52$.

15. Luo Z, Zhang S. Designer nanomaterials using ot al sel, embling peptide systems and their emerging benefit ror society. Ch, Joc Rev. 2012;41(13):4736-54

16. Liu J, Liu J, Xu H, Zhang Y, Chu L, Liu Q, et Vovel tumor-targeting, selfassembling peptide nanofiber as a or for curcumin delivery. Int J Nanomedicine. 2014;9:197-207

17. Gallagher G, Menzie S, Huan \&, Jackson yyor SN. Regional cardiac dysfunction is associated vith cific alteracrons in inflammatory cytokines and matrix metalloproteinases à cute myocardial infarction in sheep. Basic Res Cardiol. 20 2(1):63-72.

18. Radhakrishnan / rishn UM, Sethuraman S. Hydrogel based injectable scaffolds for Caro tic eration. Biotechnol Adv. 2014;32(2):449-61.

19. Skardal A, Murphy rowell K, Mack D, Atala A, Soker S. A tunable hydroge astem for lo, lerm release of cell-secreted cytokines and bioprinte wound cell delivery. J Biomed Mater Res B Appl Biomater. 2017;105(): :1986-, 000 .

20. Norouzi M, Nazari B, Miller DW. Injectable hydrogel-based drug delivery

vstems for scal cancer therapy. Drug Discov Today. 2016;21(11):1835-49.

21. Zhai M, Jiang L, Song F, Zhang B, Li J, et al. Tetrahydrocurcumin an iorates diabetic cardiomyopathy by attenuating high glucose-induced ox dative stress and fibrosis via activating the SIRT1 pathway. Oxidative Med ell Longev. 2019;2019:6746907.

2. Vamanu E, Gatea F, Sarbu I, Pelinescu D. An in vitro study of the influence of Curcuma longa extracts on the microbiota modulation process, in patients with hypertension. Pharmaceutics. 2019;11(4):191.

23. Liu R, Zhang HB, Yang J, Wang JR, Liu JX, Li CL. Curcumin alleviates isoproterenol-induced cardiac hypertrophy and fibrosis through inhibition of autophagy and activation of mTOR. Eur Rev Med Pharmacol Sci. 2018; 22(21):7500-8

24. Mokhtari-Zaer A, Marefati N, Atkin SL, Butler AE, Sahebkar A. The protective role of curcumin in myocardial ischemia-reperfusion injury. J Cell Physiol. 2018;234(1):214-22.

25. Shimizu K, Sunagawa Y, Funamoto M, Wakabayashi H, Genpei M, Miyazaki Y, et al. The synthetic curcumin analogue GO-Y030 effectively suppresses the development of pressure overload-induced heart failure in mice. Sci Rep. 2020;10(1):7172.

26. Zeng $C$, Zhong $P$, Zhao $Y$, Kanchana $K$, Zhang $Y$, Khan ZA, et al. Curcumin protects hearts from FFA-induced injury by activating Nrf2 and inactivating NF-kappaB both in vitro and in vivo. J Mol Cell Cardiol. 2015;79:1-12.

27. Bugyei-Twum A, Advani A, Advani SL, Zhang Y, Thai K, Kelly DJ, et al. High glucose induces Smad activation via the transcriptional coregulator p300 and contributes to cardiac fibrosis and hypertrophy. Cardiovasc Diabetol. 2014;13:89.

28. Pang XF, Zhang LH, Bai F, Wang NP, Garner RE, McKallip RJ, et al. Attenuation of myocardial fibrosis with curcumin is mediated by modulating expression of angiotensin $\|$ AT1/AT2 receptors and ACE2 in rats. Drug Des Devel Ther. 2015;9:6043-54.

29. Yang C, Wang Z, Ou C, Chen M, Wang L, Yang Z. A supramolecular hydrogelator of curcumin. Chem Commun (Camb). 2014;50(66):9413-5.

30. Villarino AV, Kanno Y, O'Shea JJ. Mechanisms and consequences of Jak-STAT signaling in the immune system. Nat Immunol. 2017;18(4):374-84.

31. Oshima Y, Fujio Y, Nakanishi T, Itoh N, Yamamoto Y, Negoro S, et al. STAT3 mediates cardioprotection against ischemia/reperfusion injury through metallothionein induction in the heart. Cardiovasc Res. 2005;65(2):428-35.

\section{Publisher's Note}

Springer Nature remains neutral with regard to jurisdictional claims in published maps and institutional affiliations. 